\title{
Drawing Tower In-Line Fabrication and the Spectrum Analysis of Sampled Fiber Grating
}

\author{
Yu ZHENG ${ }^{1}$, Wenjing GAO ${ }^{1}$, Haihu $\mathrm{YU}^{1 *}$, Huiyong GUO ${ }^{1}$, \\ Shan JIANG ${ }^{2}$, and Desheng JIANG ${ }^{1}$ \\ ${ }^{1}$ National Engineering Laboratory for Fiber Optic Sensing Technology, Wuhan University of Technology, Wuhan \\ 430070, China \\ ${ }^{2}$ WUTOS Technology Co., Ltd, Wuhan 430223, China \\ *Corresponding author: Haihu YUＥ-mail: hhyu@whut.edu.cn
}

\begin{abstract}
Sampled fiber grating is a special superstructure fiber Bragg grating with a wide range of applications in many fields. In this work, based on drawing tower in-line fabrication system, a new preparation method of the sampled fiber grating is proposed and experimentally demonstrated. Experimental result shows that the obtained sampled fiber gratings possess dense reflection spectra, with a minimum reflection peak interval of only $0.09 \mathrm{~nm}$. This method exhibits promising application prospect in the fabrication of the high-quality sampled fiber grating. On the other hand, the spectral characteristics of the sampled fiber grating are analyzed when the sub-grating is affected by the external physical quantities such as, in this paper, strain. Wavelength shift and intensity change in the reflection peak of the spectra indicate that the grating is affected differently by micro strains, due to the different spatial positions along the axis of the sampled fiber grating. This work is aimed at exploring the potential applications of the sampled fiber grating in quasi-distributed micro-area sensing with the millimeter level.
\end{abstract}

Keywords: Sampled fiber grating; drawing tower in-line fabrication; spectrum analysis; grating array; sensing application

Citation: Yu ZHENG, Wenjing GAO, Haihu YU, Huiyong GUO, Shan JIANG, and Desheng JIANG, "Drawing Tower In-Line Fabrication and the Spectrum Analysis of Sampled Fiber Grating," Photonic Sensors, 2020, 10(1): 80-87.

\section{Introduction}

Sampled fiber gratings (SFGs), known as special superstructure fiber Bragg gratings, have been widely researched and used in the field of multi-parameter sensing, optical filters, and multi-wavelength laser [1-6]. The SFG possesses special superstructure refractive index distribution, which is formed by multiple sub-gratings in a regular arrangement [1]. Sampled grating has a wide reflection spectrum and a narrow reflection peak, which has the advantages of high reflectivity, uniform reflection peak, and stable peak interval $[3,4]$. Currently, the main preparation methods of the sampled grating include the amplitude mask template method (a traditional method) and the point-by-point scanning method [6]. For the amplitude mask template method, the SFGs can be fabricated by using an amplitude mask on the basis of a uniform mask and have the high quality [7]. In this way, the sub-gratings can be inscribed by single laser exposure and have great consistency. However,

Received: 26 November 2018 / Revised: 10 April 2019

(C) The Author(s) 2019. This article is published with open access at Springerlink.com

DOI: $10.1007 / \mathrm{s} 13320-019-0559-4$

Article type: Regular 
this method is limited by the length and period of the amplitude mask and phase mask, then the preparation operation is not flexible. When writing different types of sampled gratings, the phase mask and the amplitude mask need to be replaced frequently. In contrast, by using the point-by-point scanning method, the sampled gratings with different parameters can be flexibly prepared by controlling the displacement distance and slit width [8]. In this way, only one sub-grating can be inscribed by each laser source, leading to that the SFGs of almost all parameters can be fabricated. So the point-by-point scanning method has a great significance in expanding the application area of the SFGs.

At present, the fiber Bragg grating (FBG) drawing tower in-line fabrication technology is capable of preparing a long array multiplexed with a large number of identical gratings [9-11]. In the process of the FBG in-line fabrication, the fiber keeps moving when the gratings are inscribed continuously, which is similar to the process of the SFGs fabrication by the point-by-point scanning method. Therefore, it is possible to fabricate the SFGs by drawing tower in-line fabrication technology. This technique can avoid the fiber decoating/recoating processes and the existence of splicing joint, which are unavoidable in the traditional FBG fabrication process. Therefore, the in-line fabrication system contributes to higher tensile strength and better applicability of the fabricated gratings [12]. However, to the best of our knowledge, no work about the in-line fabrication on drawing tower of the sampled fiber grating has been reported. In this work, we combine the point-by-point scanning method and the FBG in-line fabrication technology, then try to fabricate the sampled fiber gratings with high quality and very short peak interval by using our customized drawing tower. Furthermore, the spectral characteristics of the sampled fiber grating are analyzed in order to explore the potential applications of the SFG in quasi-distributed micro-area sensing.

\section{SFGs fabrication and results}

SFG can be regarded as a series of ultra-short FBGs arranged continuously in certain spatial intervals. Among several kinds of sampled gratings, the rectangular sampled fiber grating (RSFG) is the focus of our work, which possesses the merits of easy fabrication and high uniformity. The structure of the RSFG can be regarded as an alternate arrangement between identical sub-gratings and blank fibers. A section of blank fiber and a sub-grating form a repetitive unit, then a certain number of repetitive units combine together to form the sampled grating. Furthermore, a series of SFGs are prepared on our customized drawing tower in-line fabrication system with large capacity, uniform quality, and high production efficiency. Figure 1(a) shows the schematic diagram of this system. The in-line fabrication system writes gratings simultaneously during the fiber drawing process. Under stable drawing speed, the distance between two adjacent gratings can be precisely controlled. By adopting the point-by-point scanning method into the grating writing platform [Fig.1(b)], an in-line preparation system for the SFG is established. An ultraviolet (UV) excimer laser (ATLEX-500-I, ATL Lasertechnik) with an initial spot size of $6 \mathrm{~mm} \times 12 \mathrm{~mm}$ is used to provide single-pulse laser. After being contracted by three cylindrical mirrors and focusing onto the phase mask, the spot size is $0.7 \mathrm{~mm} \times 6 \mathrm{~mm}$ eventually. A slit is installed near before the phase mask, which can freely change the beam length to accurately control the SFG length (with error $<0.1 \mathrm{~mm}$ ). The period of the phase mask is $1073.31 \mathrm{~nm}$, and the maximum writing frequency that can be realized by the UV excimer laser is up to $500 \mathrm{~Hz}$.

Using the in-line fabrication system, three SFGs with different structural parameters are successfully fabricated. Figure 2(a) shows the experimental 
reflection spectrum of an SFG with a length of $50 \mathrm{~mm}$, a sampling period of $5 \mathrm{~mm}$, and a duty ratio of 0.1 . The laser energy is $4.5 \mathrm{~mJ}$, and the slit width is $0.5 \mathrm{~mm}$. It can be seen from the reflection spectrum that the interval between two reflective peaks is only $0.16 \mathrm{~nm}$. This interval is much narrower than that of the sampled grating prepared by the traditional method $(>0.4 \mathrm{~nm})$. By using the transfer matrix method based on the coupled-mode theory, the relationship between the reflection spectra and structure of the sampled gratings is analyzed [13-15]. Figure 2(b) shows the simulated reflection spectrum of the sampled grating with the same parameters. The experimental spectrum is basically consistent with the simulation results, proving that this system can prepare the designed sampled gratings. We further design and fabricate two sampled gratings with different parameters, and the corresponding reflection spectra are shown in Fig. 3. The sampled grating shown in Fig. 3(a) possesses the grating length of $100 \mathrm{~mm}$ and duty cycle of 0.06 , while Fig. 3 (b) shows the grating length of $80 \mathrm{~mm}$ and duty cycle of 0.05 . A narrower peak interval of $0.09 \mathrm{~nm}$ is obtained, which is in accord with the design, proving the reliability of our system.

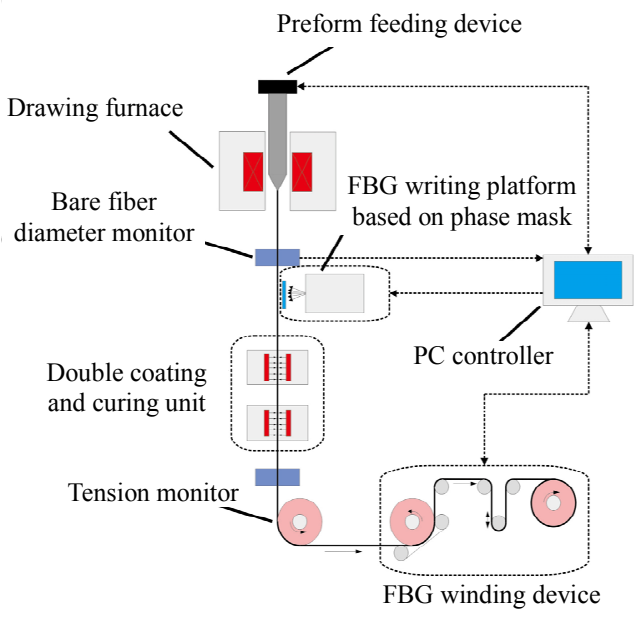

(a)

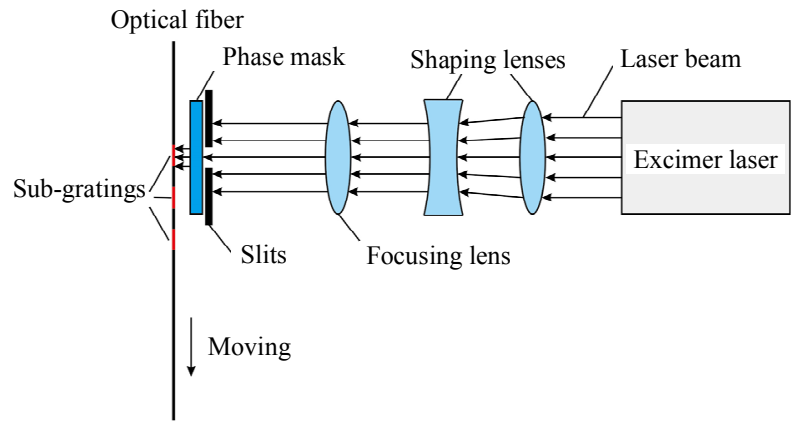

(b)

Fig. 1 Schematic diagram of (a) the FBG drawing tower in-line writing system and (b) the writing platform.

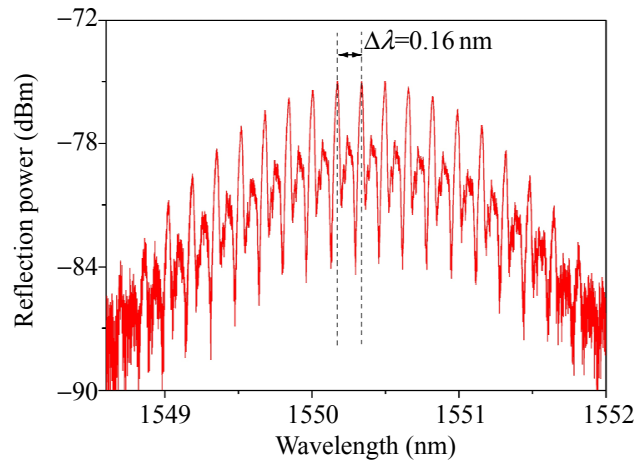

(a)

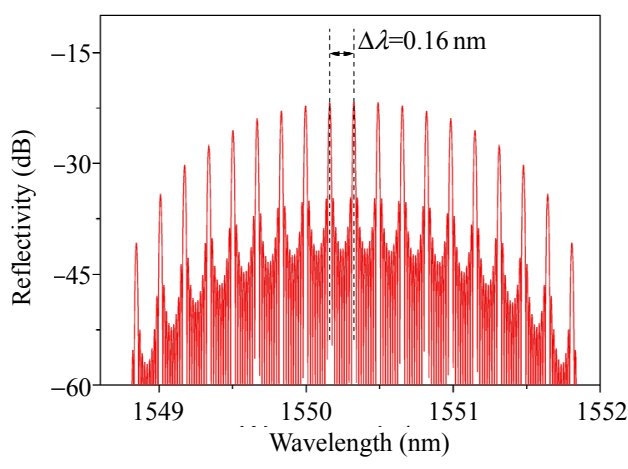

(b)

Fig. 2 Reflection spectrum (a) and the simulation spectrum (b) of the SFG prepared by the scanning method.

Moreover, two kinds of 30-meter-long SFGs arrays without any splicing joint are produced, and the interval between two adjacent SFGs is 5 meters. The fabricated arrays are then demodulated by a 
grating interrogator (LGI-100B, Sentek Instrument, U.S.A.). This grating interrogator adopting the wavelength scanning time division multiplexing (WSTDM) technique is able to demodulate SFGs with reflectivity as low as $-40 \mathrm{~dB}$. Figures 4 (a) and 4(b) show the spectra of the SFGs with the duty

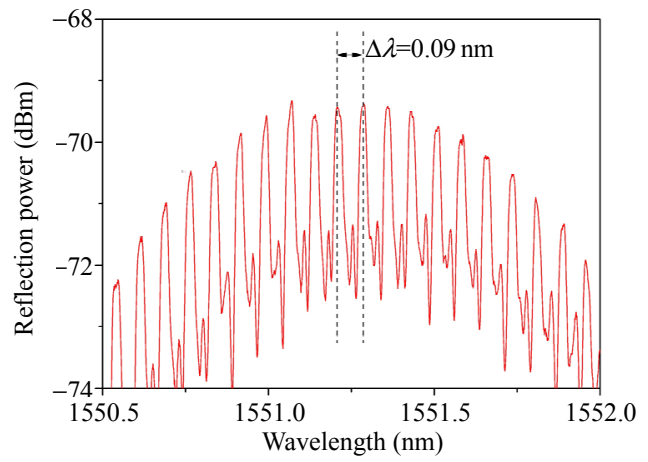

(a) cycles of 0.1 and 0.04 , respectively. The results demonstrate that fabricating SFGs array by the drawing tower is possible and executable. Therefore, based on the drawing tower in-line fabrication system, the SFGs and SFG array can be fabricated with the adjustable parameters.

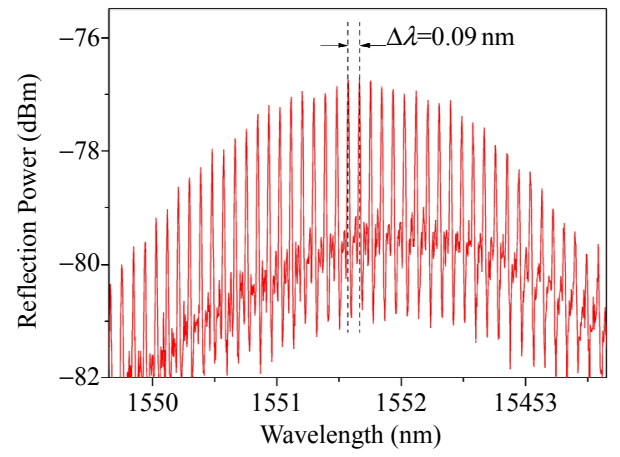

(b)

Fig. 3 Reflection spectra of SFGs prepared by the point-by-point scanning method with different parameters: (a) $a=0.6 \mathrm{~mm}$, $p=10 \mathrm{~mm}$, and $L=100 \mathrm{~mm}$; (b) $a=0.4 \mathrm{~mm}, p=8 \mathrm{~mm}$, and $L=80 \mathrm{~mm}$.

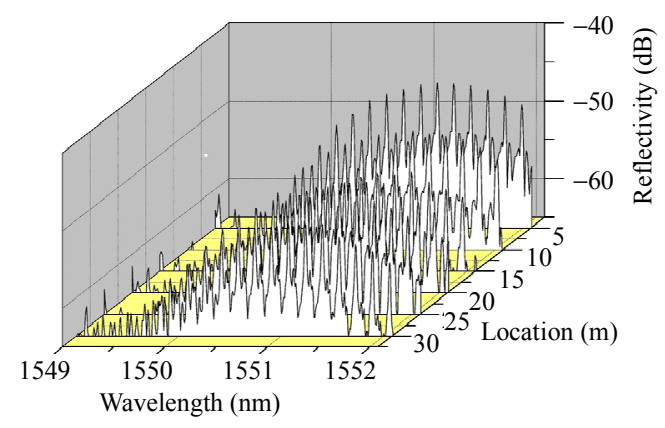

(a)

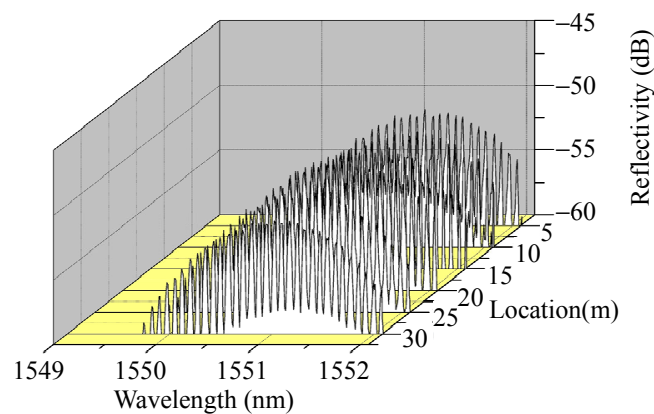

(b)

Fig. 4 Reflection spectra of SFGs array with different parameters: (a) $a=0.4 \mathrm{~mm}, p=4 \mathrm{~mm}$, and $L=40 \mathrm{~mm}$; (b) $a=0.4 \mathrm{~mm}$, $p=10 \mathrm{~mm}$, and $L=100 \mathrm{~mm}$.

\section{Simulation calculation of SFGs sensing}

For the SFGs, the change of each sub-grating will affect the spectra shape of the whole sampled grating. More importantly, when external influence causes wavelength-shift of sub-grating spectra, different spatial positions along the axis of the sub-grating will have different effects on the spectra shape of the SFG. Therefore, the positions of the sub-gratings which are affected by external environment, can be judged by observing the change in the spectra shape.

The spectra shapes of the SFG are simulated with one sub-grating under external tension. The whole sub-gratings are divided into 10 regions: 1 to
10 , depending on the position of the SFG as shown in Fig. 5. The length of the SFG is $40 \mathrm{~mm}$, and the duty cycle is 0.25 , then the reflection peak intensity and period of grating are the same as the grating shown in Fig. 2. Further, $100 \mu \varepsilon$ strain is applied to the sub-gratings at each region, respectively, and the reflection spectra of the SFG are calculated and depicted in Fig. 6. Figure 6(a) shows the reflection spectra when the SFG is not affected by strain, and Figs. 6(b) to 6(k) show the SFG reflection spectra of 10 regions: 1 to 10 , which are affected by the strain at $100 \mu \varepsilon$ separately. Due to this, a small peak is split on the right side of each reflection peak and gradually increase to the maximum, with the affected region changing from Region 1 to Region 5 . 
And then the small peak starts to decrease and eventually disappears from Region 6 to Region 10 . It shows that the sub-gratings in different areas have different influences on the reflection spectrum, and it is mainly caused by the distance from the center of the SFG. Therefore, if only considering Region 1 to Region 5, it is possible to judge the position of a sub-grating which is affected by observing the characteristics of the reflection spectra. The effect of a reflection peak on the spectral center is further compared, as shown in Fig. 7. It can be clearly seen that with the position of the sub-grating moving to the center, the original reflection peak moves to the left while a new reflection peak appears and gradually grows from the right side of the original

(a)

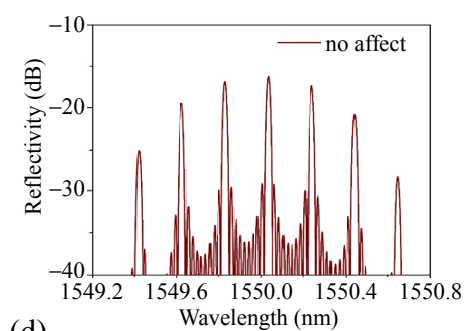

(d)

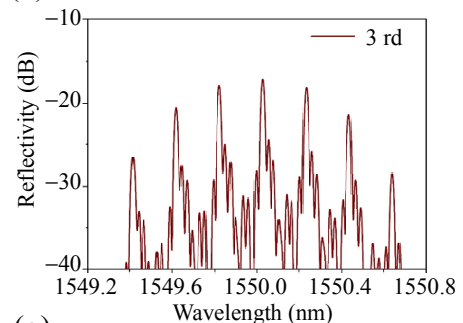

(g)

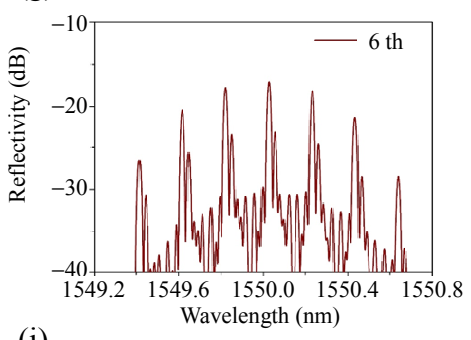

(j)

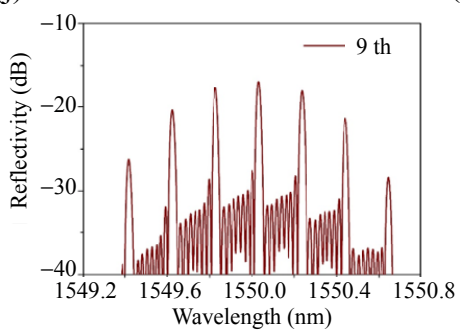

(b)

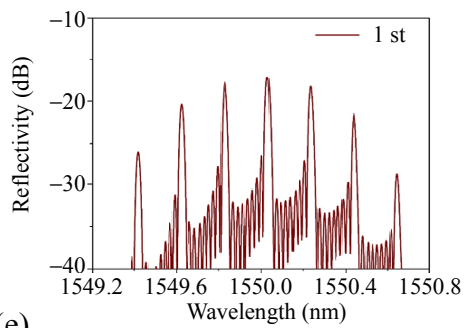

(e)

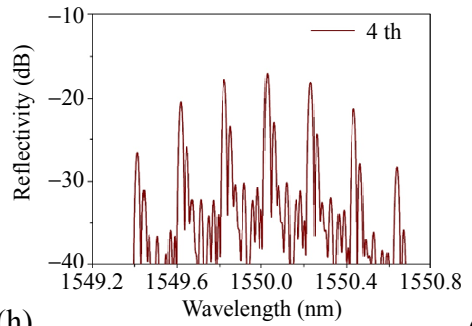

(h)

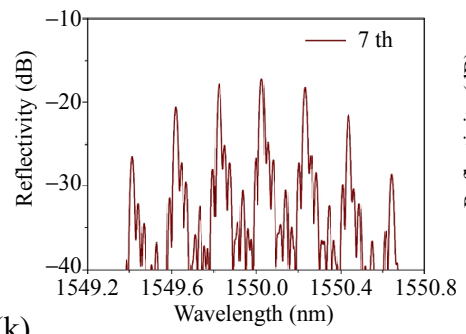

(k)

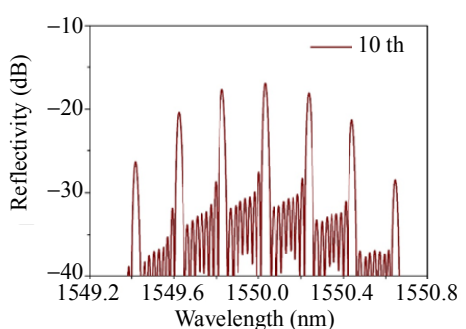

peak. This pattern of the spectral change is similar to the change rule of the phase shifted grating that the phase shift position moves from the edge to center [12]. Therefore, based on the study of the phase shift grating, the position of the affected sub-grating can be judged by the spectral change: when the peak separation in the reflection spectrum is more obvious, the position of the affected sub-grating is closer to the center.

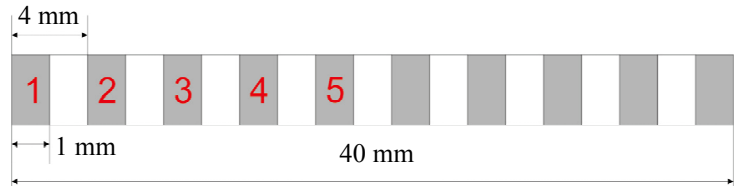

Fig. 5 Structural diagram of the SFG with parameters $a=1 \mathrm{~mm}, p=4 \mathrm{~mm}$, and $L=40 \mathrm{~mm}$.

(c)
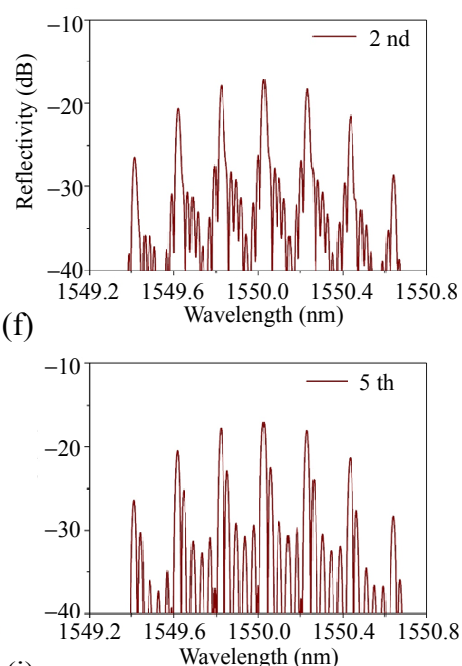

(i)

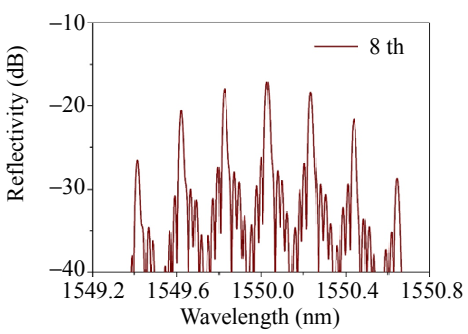

Wavelength (nm)

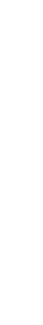

Fig. 6 Reflection spectra of the SFG with different regions of the sub-grating: (a) no affect and (b)-(k) Regions 1-10. 


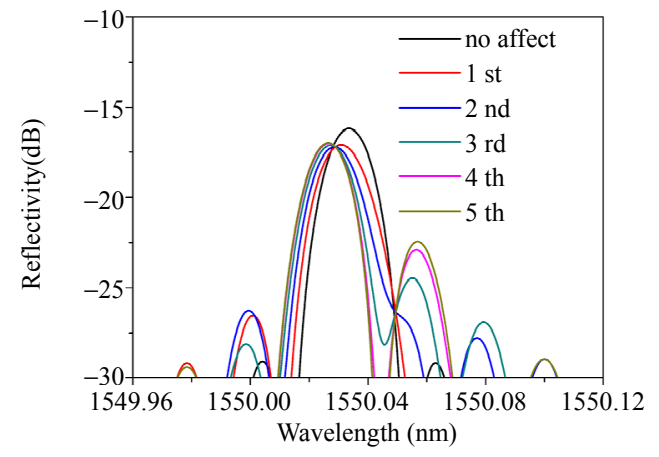

Fig. 7 Change of the reflection spectrum of the SFG with different positions of the sub-grating.

The influence of strain on the SFG reflection spectrum is further studied. A series of adjustable micro strains, rising from $100 \mu \varepsilon$ to $400 \mu \varepsilon$ with an interval of $50 \mu \varepsilon$, are applied to the sub-grating located at Region 5, and the results are shown in Fig. 8. Within this range, in the two division peaks of each original reflection peak of the SFG, the left peak keeps decreasing while the right peak keeps increasing with an increase in the strain. Figure 9 shows the variation of the central wavelength and intensity of the central reflection peak in this process. It can be seen that the two reflective peaks gradually move to the left, and the left peak gradually decreases while the right peak increases. With an increase in the applied strain, the wavelengths of the two peaks experience blue-shift linearly with the rate at $6.53 \times 10^{-5} \mathrm{~nm} / \mu \varepsilon$ and $6.89 \times 10^{-5} \mathrm{~nm} / \mu \varepsilon$ as shown in Fig. 10(a). Meanwhile, the relative reflectivity of the left peak decreases linearly at $0.0265 \mathrm{~dB} / \mu \varepsilon$, and the right peak increases at a two-order function as shown in Fig. 10(b). This study shows that by comparing the relative variation of the separation peaks, it is possible to determine the degree of strain in a sub-grating. Therefore, by using the sub-gratings on the left or right side of the SFG, the position and influence degree of the affected sub-grating can be judged by observing the variation of the reflection spectrum. (a)

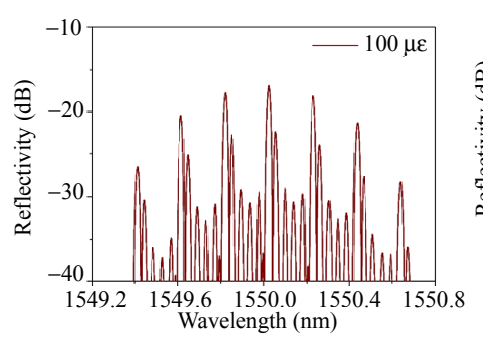

(d)

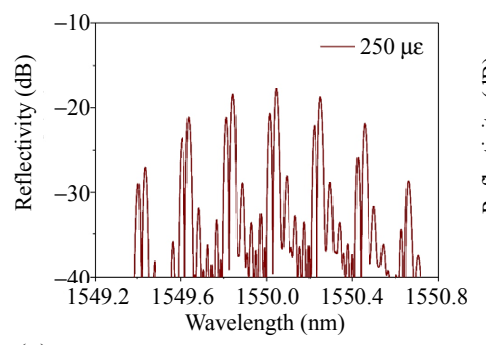

(g)

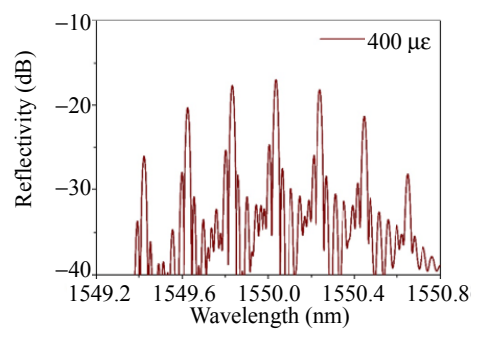

(b)

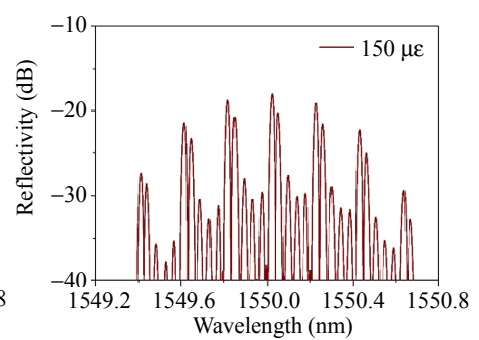

(e)

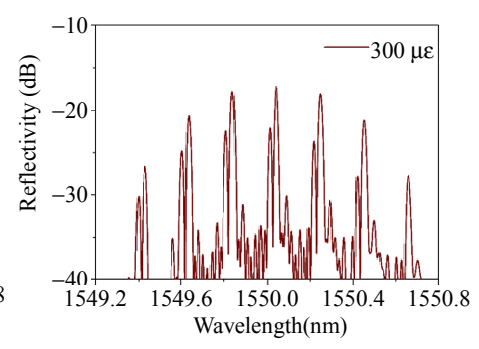

(c)

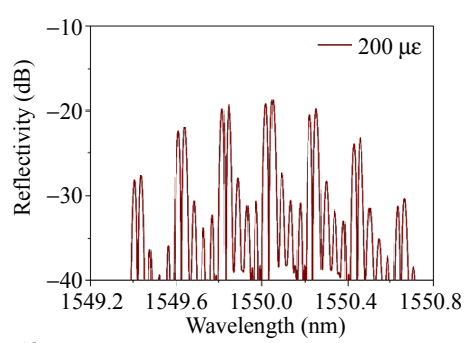

(f)

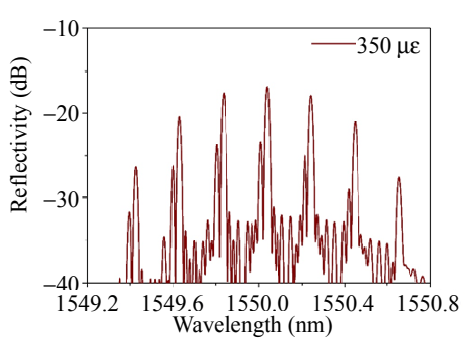

Fig. 8 Reflection spectra with different strains: (a)-(g): $100 \mu \varepsilon-400 \mu \varepsilon$. 


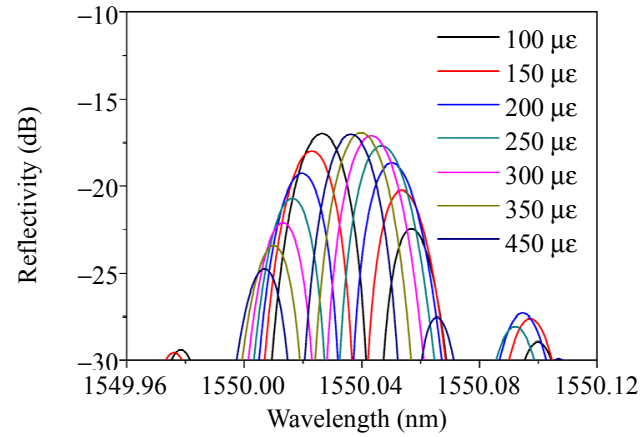

Fig. 9 Change of the central reflection peaks with different strains.

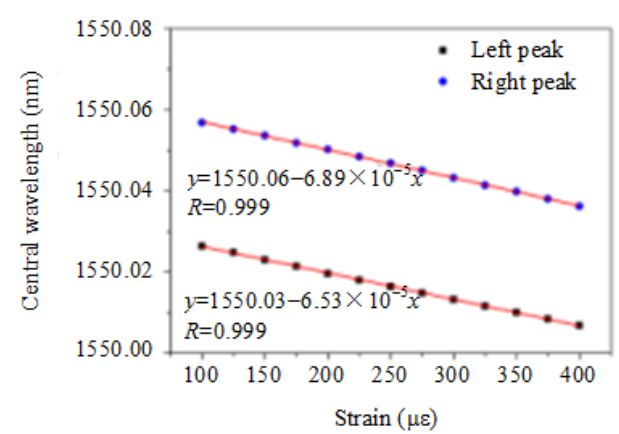

(a)

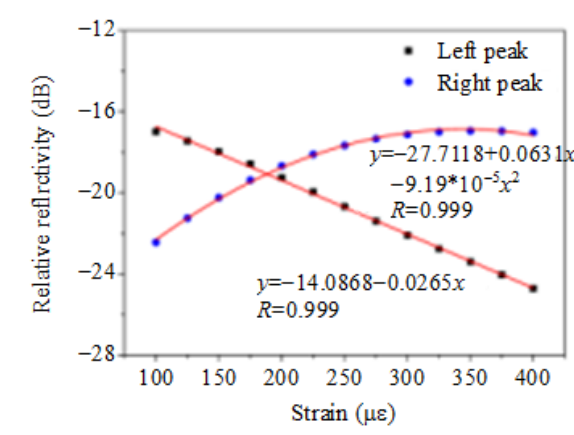

(b)

Fig. 10 Change of the reflection peaks with different strains: (a) corresponding curve of the central wavelength of two peaks to strain and (b) corresponding curve of the relative reflectivity of two peaks to strain.

\section{Conclusions}

In this paper, a new preparation method of the sampled fiber grating is proposed and experimentally demonstrated. Theoretical analysis shows that free from the grating length limitation, the sampled gratings prepared by the point-by-point scanning method possess the denser reflection peak than that of the traditionally fabricated sampled gratings. Practical fabrication is carried out by the in-line fabrication system, and three sampled gratings with much denser reflection peaks are obtained. The fabricated sampled gratings have undamaged coating layer and no splicing joint, which can enhance the applicability of the fabricated sampled grating. This method is applied to the fabrication of the SFG array with different parameters, and the reflection spectra can be measured efficiently by the grating interrogator. The simulation results show that the position and magnitude of strain in the millimeter level can be determined by the reflection spectra of the SFG. Therefore, the SFG and array can be used in the quasi-distributed micro-area sensing applications.

\section{Acknowledgment}

This work was supported by the National Natural Science Foundation of China (NSFC) (Grant Nos. 61290311 and 61735013) and the Major Scientific and Technological Innovation Project in Hubei Province (Grant No. 2015AAA001).

All authors thank the National Engineering Laboratory for Fiber Optic Sensing Technology for offering the experimental equipment.

Open Access This article is distributed under the terms of the Creative Commons Attribution 4.0 International License (http://creativecommons.org/licenses/by/4.0/), which permits unrestricted use, distribution, and reproduction in any medium, provided you give appropriate credit to the original author(s) and the source, provide a link to the Creative Commons license, and indicate if changes were made.

\section{References}

[1] W. H. Loh, F. Q. Zhou, and J. J. Pan, "Sampled fiber grating based-dispersion slope compensator," IEEE Photonics Technology Letters, 1999, 11(10): 1280-1282.

[2] O. Frazao, R. Romero, and G. Rego, "Sampled fiber Bragg grating sensors for simultaneous strain and temperature measurement," Electronics Letters, 2002, 38(14): 693-695.

[3] H. P. Li and Y. L. Sheng, "Phased-only sampled fiber Bragg gratings for high-channel-count chromatic dispersion compensation," Journal of Lightwave Technology, 2003, 21(9): 2074-2083.

[4] J. HüBner, D. Zauner, and M. Kristensen, "Strong 
sampled Bragg gratings for WDM applications," IEEE Photonics Technology Letters, 1998, 10(4): 552-554.

[5] V. Jayaraman, D. A. Cohen, and L. A. Coldren, "Demonstration of broadband tunability in a semiconductor laser using sampled gratings," Applied Physics Letters, 1992, 60(19): 23212323.

[6] A. Bergonzo, J. Jacquet, and D. de Gaudemaris, "Widely Vernier tunable external cavity laser including a sampled fiber Bragg grating with digital wavelength selection," IEEE Photonics Technology Letters, 2003, 15(8): 1144-1146.

[7] Y. Dai, X. Chen, and J. Sun, "Dispersion compensation based on sampled fiber Bragg gratings fabricated with reconstruction equivalent-chirp method," IEEE Photonics Technology Letters, 2006, 18(8): 941-943.

[8] W. Zhao, S. Liu, and H. Qi, "Sampled fiber grating for WDM signal queuing with picosecond time interval," Optics \& Laser Technology, 2017, 97: 302-307.

[9] H. Y. Guo, J. G. Tang, and X. F. Li, "On-line writing identical and weak fiber Bragg grating arrays,"
Chinese Optics Letters, 2013, 11(3): 030602.

[10] H. Y. Guo, H. H. Yu, and W. Y. Wu, "Preparation of photosensitive fibers for weak fiber Bragg grating arrays," Physics Procedia, 2013, 48: 184-190.

[11] Z. Luo, H. Wen, and H. Guo, "A time- and wavelength-division multiplexing sensor network with ultra-weak fiber Bragg gratings," Optics Express, 2013, 21(19): 799-807.

[12] Y. Zheng, H. H. Yu, and H. Y. Guo, “Analysis of the spectrum distortions of weak fiber Bragg gratings fabricated in-line on a draw tower by the phase mask technique," Journal Lightwave Technology, 2015, 33(12): 2670-2673.

[13] H. Kogelnik, "Filter response of nonuniform almost-periodic structures," The Bell System Technical Journal, 1976, 55(1): 109-126.

[14] M. Yamada and K. Sakuda, "Analysis of almost-periodic distributed feedback slab waveguides via a fundamental matrix approach," Applied Optics, 1987, 26(16): 3474-3478.

[15] H. Ke, K. S. Chiang, and J. H. Peng, "Analysis of phase-shifted long-period fiber gratings," IEEE Photonics Technology Letters, 1998, 10(11): 1596-1598. 